\title{
Adsorption Kinetics and Intraparticulate Diffusivity of Aniline Blue Dye onto Activated Plantain Peels Carbon
}

\author{
OLADAYO ADEYI*, OLUSHOLA SUNDAY AYANDA ${ }^{\mathrm{a}}$, \\ GODWIN OLADELE OLUTONA ${ }^{\mathrm{b}}$ and OLAMIDE GANIYU ${ }^{\mathrm{b}}$
}

*Department of Chemical Engineering, Faculty of Engineering, Cape Peninsula University of Technology, P.O. Box 1906, Bellville, South Africa ${ }^{\mathrm{a} D e p a r t m e n t}$ of Chemistry, Faculty of Applied Sciences, Cape Peninsula University of Technology, P.O. Box 1906, Bellville, South Africa

${ }^{\mathrm{b}}$ Department of Chemistry, Bowen University, Iwo, Osun State, Nigeria

adeyioladayo350@yahoo.com

Received 22 May 2012 / Accepted 13 June 2012

\begin{abstract}
The adsorption kinetics and intraparticulate diffusivity of aniline blue dye onto activated plantain peel carbon has been studied. Two kinetic models, the pseudo first- and second-order equations were selected to follow the adsorption process. Kinetic parameters; rate constants, equilibrium adsorption capacities and correlation coefficients, for each kinetic equation were calculated and discussed. Experimental results show that the amount of aniline blue dye adsorbed increased with increasing contact time and the adsorption rate constant $\left(\mathrm{k}_{\mathrm{ad}}\right)$ follows the second order kinetics. Results obtained also shows that the adsorption of aniline blue dye onto activated plantain peels carbon is particle-diffusion controlled.
\end{abstract}

Keywords: Plantain peels, Carbon, Adsorption kinetics, Intraparticulate diffusivities, Pseudo first order, Pseudo second order

\section{Introduction}

Synthetic dyes are used extensively by dye industries, paper industries, textile dyers, colour photography and as additives in petroleum products. The effluents thus generated by these industries are highly coloured and disposal of wastes into the environment are extremely harmful $^{1}$. The presence of synthetic dyes in water systems is unacceptable and may be visible at concentrations as low as 1 ppm thereby affecting the activity in the aquatic systems. Aniline blue dye is a synthetic dye; a mixture of methyl blue (a) and water blue (b) (Figure 1) and may also be either of them. Aniline blue dye has great harmful effect for public health and environmental quality, so more and more rigorous limits on the letting amount of aniline have been established ${ }^{2}$. Aniline blue dye is toxic, cancinogenic and the presence of the dye in natural water systems inhibits sunlight diffusion into the water, consequently 
reducing the photosynthetic process of aquatic plant. Hence, the remediation of aniline blue dye from wastewaters before discharge into the water environment is essential. In general, chemical, physical and biological treatment procedures can be used for this purpose. These individual processes differ in their efficiency to remove or degrade the dyes and also in the cost required for the treatment of the comparable volumes of polluted water. At present, adsorption has been recognized as the most popular treatment process for the removal of dye from an aqueous solution due to its simplicity, high efficiency, easy recovery and the reusability of the adsorbent ${ }^{3,4}$. Various adsorbents have been tested and used for the removal of dyes from polluted water such as activated carbon $^{5}$, Kaoline clay ${ }^{6}$ and other adsorbents ${ }^{7-9}$.<smiles></smiles>

a. Methyl blue<smiles>Cc1cc(C(=C2C=CC(=Nc3ccc(S(=O)(=O)O)cc3)C=C2)c2ccc(Nc3ccc(S(=O)(=O)O)cc3)cc2)cc([S+](=O)(=O)OC(C)(C)C)c1N</smiles>

Figure 1. Aniline blue dye

Since activated carbon is the most widely used and most effective adsorbent, its high cost tends to increase the cost of adsorption systems ${ }^{10}$. As a result, there is a search for lowcost, naturally occurring, abundant sorbent materials that can serve as viable alternatives to activated carbon. In contrast to vast reported work on the use of carbon obtained from agricultural waste for the adsorption of other types of dyes such as congo red, methylene blue etc, limited work was reported on the use of low cost adsorbents for the adsorption of aniline blue dye from wastewaters. However, Sarita and Tyagi ${ }^{11}$ reported the equilibrium and kinetic studies of the adsorption of aniline blue dye from aqueous solution onto activated rice husk carbon. They reported that aniline blue dye adsorption rate constant follows the first order kinetics.

The aim of this research work is therefore to prepare activated carbon from agricultural waste (plantain peels) and to carry out the adsorption kinetics and equilibrium studies of aniline blue dye on the activated plantain peels carbon sample. 


\section{Experimental}

The Aniline blue dye used in this study was BDH product. The stock solution of Aniline blue $\left(1000 \mathrm{mg} / \mathrm{dm}^{3}\right)$ was prepared in deionized water. Plantain peels were collected from Oyo town, Oyo State, Nigeria. The peels were dried after sand and debris have been removed. Carbonization of the sample was done by subjecting $500 \mathrm{~g}$ of the sample to low temperature conversion process in an inert atmosphere of nitrogen gas. The carbonization was carried out at two different temperatures of $360{ }^{\circ} \mathrm{C}$ and $420{ }^{\circ} \mathrm{C}$ in the presence of nitrogen to disallow oxidation of carbon. The conditions of the experiment were $1 \mathrm{~L} / \mathrm{mins}$ of Nitrogen flow and $9.7 \mathrm{~J} / \mathrm{min}$ of heating rate. The obtained carbon was ground into a particle size $\leq 0.5 \mathrm{~mm}$ carbon and was washed using de-ionised water to $\mathrm{pH} 7.10$ using $0.1 \mathrm{M} \mathrm{NaOH}$ and 0.1 M HCL.

\section{Surface modification}

Surface modification of carbon obtained from plantain peels was carried out by soaking $20 \mathrm{~g}$ of carbon in $0.1 \mathrm{M}$ nitric acid solution for three days. The solution was filtered and the residue dried in an oven at $150{ }^{\circ} \mathrm{C}$ for 24 hours.

\section{Adsorption process}

An initial aniline blue dye concentration of $1000 \mathrm{mg} / \mathrm{L}$ was prepared. $0.5 \mathrm{~g}$ of the adsorbent (activated plantain peels carbon) was thoroughly mixed with $50 \mathrm{~mL}$ aniline dye solution in a conical flask. The reaction flask, kept in a water-thermostat, was maintained at the desired constant temperature $\left(25 \pm 0.1{ }^{\circ} \mathrm{C}\right)$. Concentration of aniline blue dye solution, free of suspended carbon, at a regular interval of time was determined using a UV spectrophotometer. The amount of congo red adsorbed $(\mathrm{mg} / \mathrm{g})$ was calculated based on a mass balance equation as given below:

$$
q=\frac{(C o-C) V}{W}
$$

Where $\mathrm{q}$ is the equilibrium adsorption capacity per gram dry weight of the adsorbent, $\mathrm{mg} / \mathrm{g}$; Co is the initial concentration of aniline blue dye in the solution, $\mathrm{mg} / \mathrm{L}$; $\mathrm{C}$ is the final or equilibrium concentration of aniline blue dye in the solution, $\mathrm{mg} / \mathrm{L}$; $\mathrm{V}$ is the volume of the solution, $\mathrm{L}$ and $\mathrm{W}$ is the dry weight of the adsorbent, $\mathrm{g}$.

\section{Adsorption kinetics and intraparticle diffusivity}

To analyze the adsorption rates of aniline blue dye onto activated plantain peels carbon, two simple kinetic models were tested. The models are pseudo first-order and pseudo secondorder models. The plot of either of the two models that gave better fit to the experimental data i.e. high correlation coefficient $\left(\mathrm{R}^{2}\right)$ will be chosen as the kinetic model that best described the adsorption aniline blue dye onto activated plantain peels carbon. The rate of attainment to equilibrium may also be film-diffusion controlled or particle-diffusion controlled $^{12}$. The linear driving force concept was thus used to develop the relationship for particle-diffusion controlled adsorption.

\section{Results and Discussion}

\section{Adsorption studies}

Experimental results of the adsorption of aniline blue dye onto activated plantain peels carbon (Figure 2) shows that the amount of aniline blue adsorbed increase with increasing contact time between 10-40 min until the amount of aniline blue dye adsorbed tends to a plateau at 50-60 min contact time. 


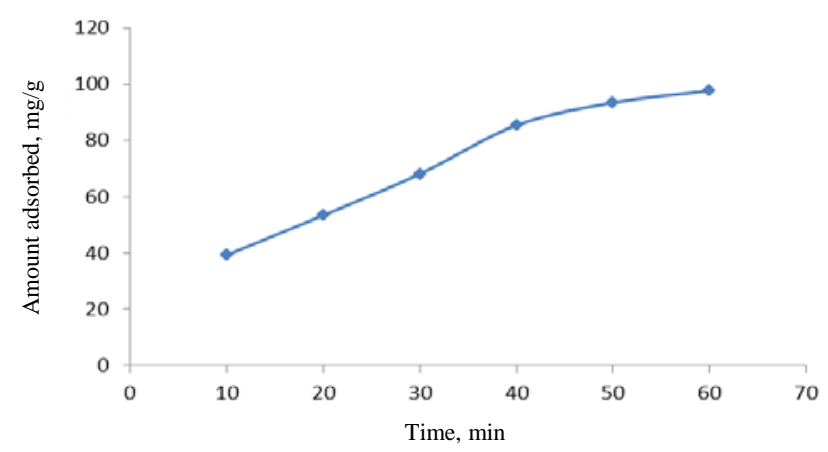

Figure 2. Amount adsorbed (mg/g) against time (min)

About $98.0 \%$ aniline blue dye was adsorbed at 60 min contact time. The obtained data was further analyzed using pseudo first-order and pseudo second-order models.

\section{Adsorption kinetics}

\section{Pseudo first-order model}

The pseudo first-order equation is generally expressed as follows:

$$
\mathrm{d}_{\mathrm{q}} / \mathrm{d}_{\mathrm{t}}=\mathrm{k}_{\mathrm{ad}}\left(\mathrm{q}_{\mathrm{e}}-\mathrm{q}_{\mathrm{t}}\right)
$$

Where, $q_{e}$ is the amount of aniline blue dye adsorbed at equilibrium per unit weight of the adsorbent (mg/g); $\mathrm{q}_{\mathrm{t}}$ is the amount of aniline blue dye adsorbed at any time $(\mathrm{mg} / \mathrm{g})$ and $\mathrm{k}_{\mathrm{ad}}$ is the pseudo first-order rate constant/min. After integration and applying boundary conditions $\mathrm{t}=0$ to $\mathrm{t}=\mathrm{t}$ and $\mathrm{q}_{\mathrm{t}}=0$ to $\mathrm{q}_{\mathrm{t}}=\mathrm{q}_{\mathrm{t}}$, the integrated form of Equation (2) becomes:

$$
\log \left(\mathrm{q}_{\mathrm{e}}-\mathrm{q}_{\mathrm{t}}\right)=\log \mathrm{q}_{\mathrm{e}}-\mathrm{k}_{\mathrm{ad}} \mathrm{t} / 2.303
$$

The values of $\log \left(\mathrm{q}_{\mathrm{e}}-\mathrm{q}_{\mathrm{t}}\right)$ were linearly correlated with $\mathrm{t}$. The plot of $\log \left(\mathrm{q}_{\mathrm{e}}-\mathrm{q}_{\mathrm{t}}\right)$ versus $\mathrm{t}$ should give a linear relationship from which $\mathrm{k}_{\mathrm{ad}}$ and $\mathrm{q}_{\mathrm{e}}$ can be determined from the slope and intercept of the plot respectively. Figure 3 shows the pseudo first-order rate equation plot for aniline blue dye adsorption onto activated plantain peels carbon.

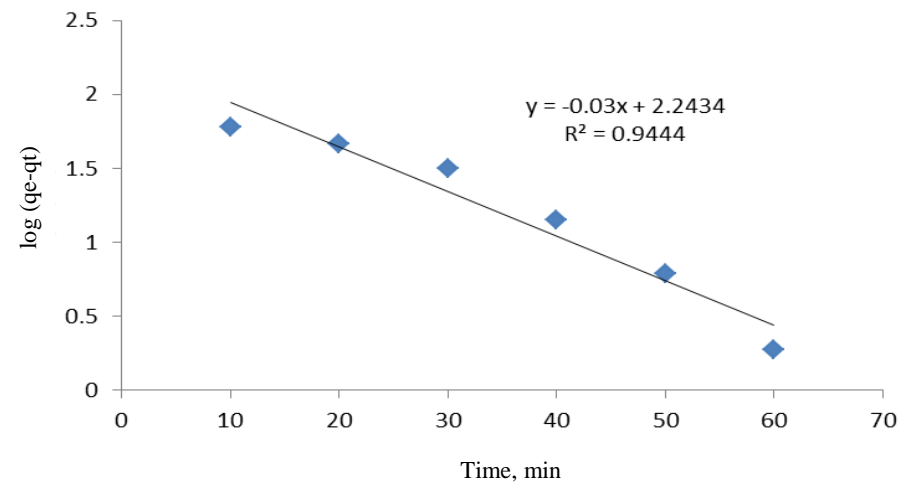

Figure 3. Pseudo first-order rate equation plot for aniline blue dye adsorption on activated plantain peels carbon

\section{Pseudo second-order model}

The pseudo second-order adsorption kinetic rate equation is expressed as ${ }^{13}$ :

$$
\mathrm{d}_{\mathrm{q}} / \mathrm{d}_{\mathrm{t}}=\mathrm{k}_{2}\left(\mathrm{q}_{\mathrm{e}}-\mathrm{q}_{\mathrm{t}}\right)^{2}
$$


Where, $\mathrm{k}_{2}$ is the rate constant of pseudo second-order adsorption (g/mg min). For the boundary conditions $\mathrm{t}=0$ to $\mathrm{t}=\mathrm{t}$ and $\mathrm{q}_{\mathrm{t}}=0$ to $\mathrm{q}_{\mathrm{t}}=\mathrm{q}_{\mathrm{t}}$, the integrated form of Equation (3) becomes:

$$
\mathrm{t} / \mathrm{q}=1 / \mathrm{h}_{\mathrm{o}}+1 / \mathrm{q}_{\mathrm{e}}(\mathrm{t})
$$

Where, $h_{o}$ is the initial adsorption rate. If the second-order kinetics is applicable, the plot t/q against $t$ in Equation (5) should give a linear relationship from which the constants $\mathrm{q}_{\mathrm{e}}$ and $\mathrm{h}_{\mathrm{o}}$ can be determined. When $\mathrm{t}$ tends to $0, \mathrm{~h}_{\mathrm{o}}$ is defined $\mathrm{as}^{14}$ :

$$
\mathrm{h}=\mathrm{k}_{2} \mathrm{q}_{\mathrm{e}}^{2}
$$

$\mathrm{K}_{2}$ was calculated for the adsorption of aniline blue dye onto activated plantain peels carbon. Figure 4 thus shows the pseudo second-order rate equation plot for aniline blue dye adsorption onto activated plantain peels carbon.

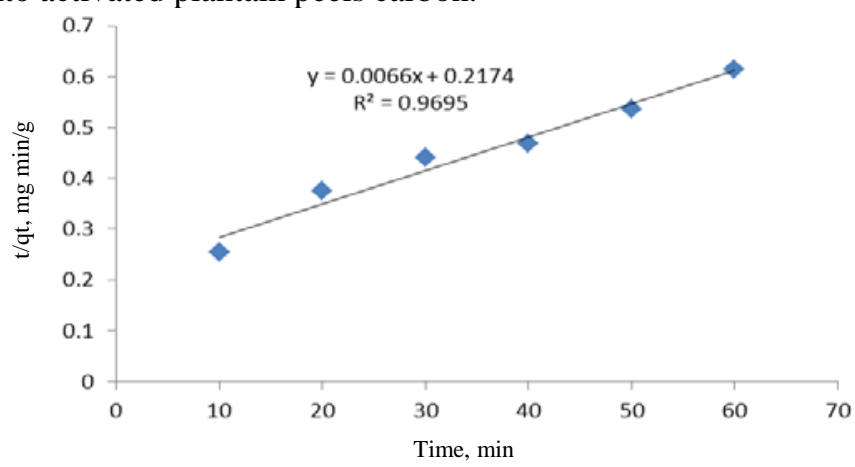

Figure 4. Pseudo second-order rate equation plot for aniline blue dye adsorption on activated plantain peels carbon

Comparing the correlation coefficient $\left(\mathrm{R}^{2}\right)$ of pseudo first- and second-order rate equation plots (Table 1), pseudo second-order rate equation plot have the highest coefficient, hence, the process of adsorption is pseudo second-order.

Table 1. Pseudo first- and second-order rate equation constants and correlation coefficient $\left(\mathrm{R}^{2}\right)$ for the adsorption of aniline blue dye onto activated plantain peels carbon

\begin{tabular}{cccc}
\hline \multicolumn{3}{c}{ Pseudo first-order } \\
\hline $\mathrm{q}_{\mathrm{e}}$ & $\mathrm{k}_{\mathrm{ad}}$ & $\mathrm{R}^{2}$ \\
175.15 & \multicolumn{2}{c}{$6.91 \times 10^{-2}$} & 0.9444 \\
Pseudo second-order & \multicolumn{3}{c}{} \\
$\mathrm{q}_{\mathrm{e}}$ & $\mathrm{k}_{2}$ & $\mathrm{~h}_{\mathrm{o}}$ & $\mathrm{R}^{2}$ \\
151.52 & $2.0 \times 10^{-4}$ & 4.5994 & 0.9695 \\
\hline
\end{tabular}

\section{Removal efficiency and fractional attainment to equilibrium}

The removal efficiency $(\mathrm{E})$ of aniline blue dye on the activated plantain peels carbon can be calculated from Equation (7) ${ }^{15}$ while the fractional attainment to equilibrium $(\alpha)$ was also used to analyze the equilibrium (Equation 8).

$$
\begin{aligned}
\mathrm{E} & =\mathrm{C}_{\mathrm{t}} / \mathrm{C}_{\mathrm{o}} \\
\alpha & =\mathrm{C}_{\mathrm{t}} / \mathrm{C}_{\infty}
\end{aligned}
$$

Where, $C_{t}$ is the concentration of aniline blue dye at any time $t, C_{o}$ is the initial concentration and $\mathrm{C}_{\infty}$ is the concentration at equilibrium. The plot of $\alpha$ against time (Equation 7) 
for the adsorption of aniline blue dye onto activated plantain peels carbon was reported. The removal efficiency (E) of aniline blue dye on the activated plantain peels carbon was presented in Table 2.

Table 2. Fraction of the amount of adsorbed aniline blue dye on activated plantain peels carbon

\begin{tabular}{cc}
\hline Time, min & $\mathrm{C}_{\mathrm{t}} / \mathrm{C}_{\mathrm{o}}$ \\
\hline 10 & 0.3922 \\
20 & 0.5336 \\
30 & 0.6810 \\
40 & 0.8534 \\
50 & 0.9341 \\
60 & 0.9764 \\
\hline
\end{tabular}

The plot of $\alpha$ (fractional attainment to equilibrium) against time for the adsorption of aniline blue dye on the activated plantain peels carbon is as shown in Figure 5.

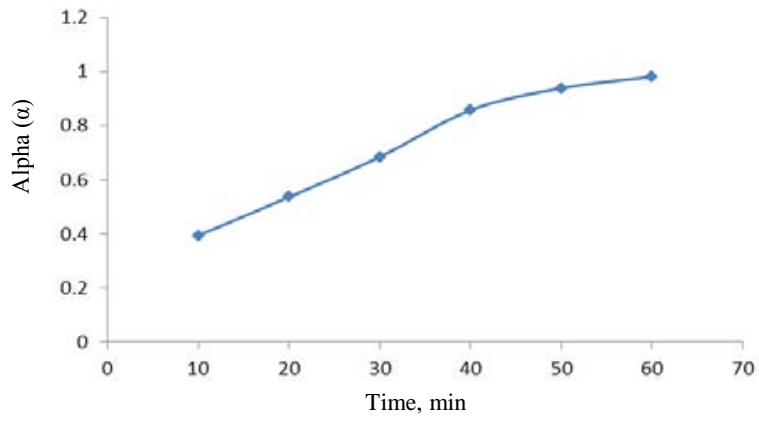

\section{Intraparticle diffusivity}

Figure 5. Alpha $(\alpha)$ against time (min)

The linear driving force concept was used to develop the relationship for particle-diffusion controlled adsorption as shown by Equation (8).

$$
\ln (1-\alpha)=-\mathrm{K}_{\mathrm{p}} \mathrm{t}
$$

$K_{p}$ is the rate coefficient for particle-diffusion controlled process corresponding to the particle size of the adsorbent; $t$ is time and $\ln (1-\alpha)$ is a measure of the intra-particulate diffusivity. If a plot of ln (1- $\alpha$ ) versus time results in a linear relationship, the adsorption process is particle-diffusion controlled and the diffusivity of aniline blue dye onto the adsorbent surface is independent of the extent of adsorption.

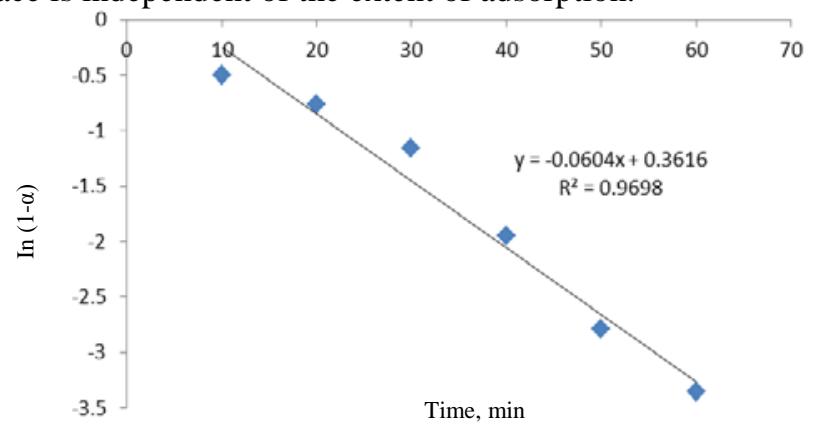

Figure 6. Intraparticle diffusivity plot for aniline adsorption on activated plantain peels carbon 
As shown from Figure 6, it was observed that the adsorption process tested was particle diffusion controlled. The values of $K_{p}$ from the slope of the graph and the coefficient of regression $\left(\mathrm{R}^{2}\right)$ are $6.04 \times 10^{-2}$ and 0.9698 respectively.

\section{Conclusion}

The results obtained clearly suggest that activated plantain peels carbon can be efficiently used for the removal of aniline blue dye. About $98.0 \%$ aniline blue dye was adsorbed at 60 min contact time and the adsorption rate constant $\left(\mathrm{k}_{\mathrm{ad}}\right)$ follows the second order kinetics. The use of activated plantain peels carbon to clean up aniline blue dye bearing effluents from textile industries is cheap, feasible and environment-friendly, helps to keep the environment clean and will also provide an affordable technology for small and medium scale industries.

\section{References}

1. Benaissa H, Int J Environ Pollut., 2008, 34(1-4), 71-82.

2. An F, Feng X and Gao B, J Hazard Mater., 2010, 178(1-3), 499-504.

3. Garg V K, Gupta R, Yadav A B and Kumar R, Bioresource Technol., 2003, 89(2), 121-124.

4. Aksu Z, Process Biochem., 2005, 40, 997-1026.

5. Han Y, Quan X, Chen S, Zhao H, Cui C and Zhao Y, Sep Purif Technol., 2006, 50, 365-372.

6. Unuabonah E I, Adebowale K O and Dawodu F A, J Hazard Mater., 2008, 157(2-3), 397-409.

7. Eimer G A, Costa M G, Pierella L B and Anunziata O A, J Colloid Interface Sci., 2003, 263, 400-407.

8. Z Zheng H, Liu D, Zheng Y, Liang S and Liu Z, J Hazard Mater., 2009, 167, 141-147.

9. Lin X, Zhang J, Luo X, Zhang C and Zhou Y, Chem Eng J., 2011, 172, 856-863.

10. Kapdan I K, Kargi F, McMullan G and Marchant R, Environ Technol., 2000, 21(2), 231-236.

11. Sarita Y, Tyagi D K and Yadav O P, Int J Chem Res., 2011, 2(3), 59-64.

12. Abia A A and Igwe J C, Afr J Biotech., 2005, 4(6), 509-512.

13. Ho Y S, McKay G, Wase D A J and Foster C F, Adsorp Sci Technol., 2000, 18, 639-650.

14. Horsfall M and Spiff A I, Electron J Biotech., 2004, 7(3), 1-7.

15. Saifuddin M N and Kumaran P, Electron J Biotech., 2005, 8(1), 1-7. 